\title{
Photodynamic action of protoporphyrin IX derivatives on Trichophyton rubrum*
}

\author{
Rogério Rodrigo Ramos ${ }^{1,2}$ \\ Adjaci Uchôa Fernandes ${ }^{2,3}$
}

\author{
Dora Inês Kozusny-Andreani ${ }^{2}$ \\ Mauricio da Silva Baptista ${ }^{3}$
}

DOI: http:/ / dx.doi.org/10.1590/abd1806-4841.20163643

\begin{abstract}
BACKGROUND: Dermatophytes are filamentous keratinophilic fungi. Trichophyton rubrum is a prevalent infectious agent in tineas and other skin diseases. Drug therapy is considered to be limited in the treatment of such infections, mainly due to low accessibility of the drug to the tissue attacked and development of antifungal resistance in these microorganisms. In this context, Photodynamic Therapy is presented as an alternative.

OвJестіVE: Evaluate, in vitro, the photodynamic activity of four derivatives of Protoporphyrin IX by irradiation with LED $400 \mathrm{~nm}$ in T. rubrum.

METHOD: Assays were subjected to irradiation by twelve cycles of ten minutes at five minute intervals.

RESULT: Photodynamic action appeared as effective with total elimination of UFCs from the second irradiation cycle. CONCLUSION: Studies show that the photodynamic activity on Trichophyton rubrum relates to a suitable embodiment of the photosensitizer, which can be maximized by functionalization of peripheral groups of the porphyrinic ring. Keywords: Photosensitizing agents; Tinea; Trichophyton
\end{abstract}

\section{INTRODUCTION}

Dermatophytes are filamentous keratinophilic fungi, imperfect, taxonomically related, which presents capacity to invade keratinized tissues of men and animals, causing dermatophytosis or tinea, a disease with a contagious nature and high worldwide prevalence. ${ }^{1}$ These fungi have an enzymatic system essential to metabolize keratin, used as a substrate for obtaining nutrients and energy. Dermatophytes are classified into three kinds: Microsporum, Trichophyton and Epidermophyton, and Trichophyton rubrum ( $T$. rubrum) is the prevalent infectious agent in tineas and other skin diseases, which may be persistent..$^{2-5}$

Infections caused by T. rubrum in humans are associated with failure of the host's immune response, or to the pathogen's ability to evade the defense sys- tem, thus remaining in the superficial skin layers. ${ }^{6}$ Other factors directly related to T. rubrum are the cell wall components, such as galactomannans, which may present immunosuppressive effects, and the membrane transport proteins, which are important virulence factors that ensure successful colonization in propitious environments. ${ }^{7,8}$ In the infection process, the cell wall of the dermatophyte plays an essential role, and is therefore a target to antifungal. ${ }^{9}$

Tinea treatment, in general, consists of fungicidal or fungistatic drugs of topical or systemic use, which should act specifically against the pathogenic agent, thus avoiding adverse events to the host. ${ }^{6}$ Topical treatment is satisfactory when lesions are mild. However, in the case of persistent tineas, systemic

Received on 22.04.2014.

Approved by the Advisory Board and accepted for publication on 26.04.2015.

Study performed at Laboratório de Microbiologia of the Universidade Camilo Castelo Branco (Unicastelo) and Universidade de São Paulo (USP) - São Paulo (SP), Brazil.

Financial support: Fapesp, project Fotossensibilização nas ciências da vida, process number 12/50680-5 and REDOXOME - Center for Research on Redox Processes in Biomedicine. Process number 2013/07937-8

Conflict of interest: None.

Fundação Educacional de Fernandópolis (FEF) - Fernandópolis (SP), Brazil

Universidade Camilo Castelo Branco - Fernandópolis (SP), Brazil.

Universidade de São Paulo (USP) - São Paulo (SP), Brazil.

(C2016 by Anais Brasileiros de Dermatologia 
therapy is employed. In these cases it is necessary to take into consideration the risks of drug interactions and hepatotoxicity, which can be serious. ${ }^{5}$ These treatment options frequently result in failure, since the commonly used antifungal agents have limited cellular targets. Moreover, emergence of resistant strains occurs, concomitant with high costs treatments for long periods, with possible adverse events, as well as drug application difficulty and disease relapses after discontinuation of antifungal therapy. ${ }^{6,10}$

Difficulties in the treatment of tinea by conventional methods have led to the search for safer, more efficient, easily achievable and cost-effective antifungal therapy. While some drugs produce effects only to metabolically active and/or just fungiostatic parasites, photodynamic therapy (PDT) consistis in the destruction of living tissue by visible light in the presence of a photosensitizer (PS) and molecular oxygen. PDT causes demise both in metabolically active cells, such as yeast and hyphae, as well as in resistant forms, such as conidia. ${ }^{11}$

After the discovery of penicillin, antibiotics have been occupying a central role in combating microorganisms. ${ }^{12,13}$ Since then, studies with PDT were directed to other therapies, such as cancer. This was due to the fact that antibiotics have been presented as the ultimate solution to combat microorganisms. After the discovery of development of microorganisms resistance to antibiotics, PDT rises again as an alternative. This therapy has been studied in the fight against Trichophyton rubrum and other dermatophytes. ${ }^{14,15}$ It was also shown that PDT activity is related to the type of PS, the intensity of the incident radiation and the $\mathrm{pH}$ of the medium. ${ }^{5}$ PDT, when applied under appropriate conditions, can provide photo oxidation of hyphae and conidia and total elimination of these patogens. ${ }^{5}$ However, the therapy development demands studies that provide greater selectivity and hence greater activity in combating pathogenic microorganisms.

In this study we assessed the activity of PS derived from protoporphyrin IX, aiming at incorporating strategies to fight Trichophyton rubrum.

\section{METHODS}

Biological material: Trichophyton rubrum ATCC (American Type Culture Collection) 28188 strain was used, which was grown for seven days on Sabouraud Dextrose Agar (SDA, OXOID ${ }^{\circledR}$ ), using incubation at $28^{\circ} \mathrm{C}$.

Photosensitizers: Tested PS (Pp IX dimethyl ester, PpNpNA and PpNpNI) was obtained by derivatization of protoporphyrin IX, through procedure analogue to the one described in the literature. Structural characterization was performed by mass spectrome- try and nuclear magnetic resonance of hydrogen, by one-dimensional and two-dimensional techniques at $300 \mathrm{MHz}$ and $500 \mathrm{MHz}$. As reference, Hp IX was tested, obtained from Sigma-Aldrich. For the tests, PS were dissolved in dimethylsulfoxide (DMSO) at a concentration of $1 \mathrm{mg} / \mathrm{mL}^{-1}$, and subsequently diluted in $\mathrm{NaCl}$ solution $(0.5 \%)$ for the test concentration $[20 \mu \mathrm{M}]$.

In vitro test: T. rubrum ATCC 28188 was cultured for 7 days, amid tryptone soya broth (TSB OXOID $\left.^{\circledR}\right)$, centrifuged (4000 rpm) for 5 minutes and the supernatant was discarded. The material was resuspended in sterile $\mathrm{NaCl}(0.5 \%)$ and subjected to further centrifugation. This procedure was repeated 5 times, in order to eliminate the components of the culture medium. At the end of the cycle, the material was resuspended in $1 \mathrm{ml}$ of sterile $\mathrm{NaCl}(0.5 \%)$ and stirred vigorously for 1 minute. Then, $9 \mathrm{ml}$ of $\mathrm{NaCl}$ was added and the resulting solution was subjected to stirring for 1 minute and adjusted to a McFarland standard tube 0.5 , which corresponds to approximately $1.5 \times 10^{8}$ CFU mL ${ }^{-1}$; this solution was diluted 100 times for the tests, resulting in concentration of $1.5 \times 10^{6} \mathrm{CFU} \mathrm{ml}{ }^{-1}$.

Antifungal activity: Experimental design was completely randomized, comprising 6 treatments: 1) control without application of light and PS; 2) application of light; 3) PS (Hp IX, Pp IX Dimethyl Ester, $\mathrm{PpNpNA}, \mathrm{PpNpNI}$, ) and irradiated LED $\approx 400 \mathrm{~nm}$. The experiment was conducted in vitro in from November to December 2013 and January 2014. For the tests, $1,1 \mathrm{~mL}$ of the fungal suspension and $0,05 \mathrm{~mL}$ of the PS solution were added to a centrifuge tube with $2 \mathrm{~mL}$ capacity, where it was incubated for 15 minutes at room temperature and protected from light. After incubation, samples were subjected to 12 cycles of 10 minutes of irradiation with LED at $400 \mathrm{~nm}$ and 21 $\mathrm{mW}$ power. Cycles were interspersed with a period of 5 minutes. At each cycle, $0,05 \mathrm{~mL}$ was removed and transferred to a petri dish containing Sabourad Dextrose Agar. The medium was uniformly distributed with the aid of sterile glass beads, and the plates were incubated at $28^{\circ} \mathrm{C}$ for 7 days. After this time, colony count was performed for the assessment of photodynamic activity against $T$. rubrum. The whole procedure was performed in triplicate.

Incorporation Studies: The study was carried out by incorporation of fluorescence microscopy. To obtain images, T. rubrum was grown in TSB medium at room temperature as described above.

Preparation of the sample: In a $2 \mathrm{ml}$ capacity centrifuge tube, $1 \mathrm{ml}$ of suspension at a concentration of $1.5 \times 10^{6} \mathrm{CFU} \mathrm{mL}-1$ and $100 \mu \mathrm{L}$ of PS solution were 
added, at the concentration of $20 \mu \mathrm{M}$, which was prepared in saline $\mathrm{NaCl} 0.5 \%$. The sample was homogenized and incubated for 15 minutes. For the preparation of the blades, an aliquot was removed from the sample, placed on slides and coated with cover slips. Images were obtained in a fluorescence microscope, Zeiss Axiovert 200, with objective lens of $40 \mathrm{X}$. The excitement was held in blue $(470 \pm 20 \mathrm{~nm})$ and green (546 $\pm 12 \mathrm{~nm}$ ), and the emission was recorded for the higher $\lambda 515 \mathrm{~nm}$ and $590 \mathrm{~nm}$, respectively. Images were recorded on a Canon Power Shot G10 camera.

Statistical analysis: Data were tabulated and submitted to analysis of variance (ANOVA) and means were compared using Bonferroni test with 5\% significance level.

\section{RESULTS}

Collection of photosensitizers: PS were obtained by derivatization of protoporphyrin IX via acid chloride and nucleophilic substitution with a corresponding amine or by Fisher esterification. Structural characterization, as well as the incomes, were similar to that described by Uchôa et al. ${ }^{16}$ Compounds structures are shown in figure 1.
Photodynamic activity in Trichophyton rubrum: Photodynamic action of Protoporphyrin IX derivatives on Trichophyton rubrum ATCC 28188 radiated at $400 \mathrm{~nm}$ is presented in table 1 .

Table 1 shows that the control (CT) presented a mean growth between 3206 and 3370 CFU. When the sample was irradiated $(\mathrm{L}+)$, a significant decrease was observed, and this decrease evolved over time for the first 6 cycles. In the tests with irradiation in the presence of a PS, a marked reduction from the first irradiation cycle was observed.

For a better visualization, results were transformed to percentage of reduction and are shown in figure 2, thereby providing a better asssess between the antifungal potential of each photosensitizer.
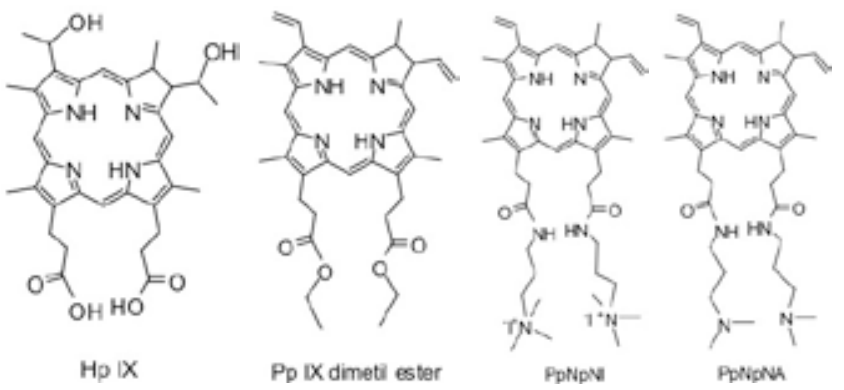

FigurE 1: Chemical structure of photosensitizers derived from protoporphyrin IX

TABLE 1: Photodynamic activity of protoporphyrin IX derivates on Trichophyton rubrum ATCC 28188; values expressed by the arithmetic mean of colony forming units

\begin{tabular}{lllllll}
\hline \multirow{2}{*}{$\begin{array}{l}\text { IRRADIATION } \\
\text { (Minutes) }\end{array}$} & \multicolumn{5}{c}{ PHOTOSENSITIZERS } \\
\cline { 2 - 6 } & $\mathrm{CT}$ & $\mathrm{L}+$ & $\mathrm{Hp}$ IX+L+ & $\begin{array}{c}\text { Pp IX dimethyl } \\
\text { ester }+\mathrm{L}+\end{array}$ & PpNpNI+L+ & PpNpNA+L+ \\
\hline 10 & $3253.33 \pm 83.26$ & $2143.33 \pm 113.70$ & $30 \pm 4.35$ & $177 \pm 51.97$ & $9.66 \pm 16.74$ & $405.33 \pm 317.67$ \\
20 & $3295 \pm 144.63$ & $2106.66 \pm 230.34$ & $23.66 \pm 8.02$ & $127.66 \pm 13.61$ & $1.33 \pm 2.30$ & $18.66 \pm 31.46$ \\
30 & $3298.66 \pm 90.53$ & $2005.33 \pm 201.23$ & $18.33 \pm 2.08$ & $64.33 \pm 37.28$ & $0 \pm 0$ & $0.33 \pm 0.57$ \\
40 & $3206 \pm 130.95$ & $1821.66 \pm 186.15$ & $31 \pm 14$ & $15.66 \pm 12.70$ & $0 \pm 0$ & $0 \pm 0$ \\
50 & $3261 \pm 145.81$ & $1940 \pm 172.86$ & $25.33 \pm 8.38$ & $7.66 \pm 7.37$ & $0 \pm 0$ & $0 \pm 0$ \\
60 & $3308.33 \pm 190.54$ & $1880 \pm 84.78$ & $23.33 \pm 4.93$ & $2 \pm 2.64$ & $0 \pm 0$ & $0 \pm 0$ \\
70 & $3252 \pm 176.44$ & $1605 \pm 111.44$ & $23 \pm 9.53$ & $0.33 \pm 0.57$ & $0 \pm 0$ & $0 \pm 0$ \\
80 & $3316.66 \pm 98.09$ & $1843.66 \pm 122.31$ & $21.66 \pm 4.93$ & $1.66 \pm 2.08$ & $0 \pm 0$ & $0 \pm 0$ \\
90 & $3341.66 \pm 63.90$ & $1637 \pm 445.48$ & $16.66 \pm 1.52$ & $0.33 \pm 0.57$ & $0 \pm 0$ & $0 \pm 0$ \\
100 & $3370.33 \pm 74.46$ & $1806.66 \pm 176.13$ & $20.66 \pm 5.85$ & $1.33 \pm 2.30$ & $0 \pm 0$ & $0 \pm 0$ \\
110 & $3355 \pm 61.44$ & $1833.33 \pm 37.85$ & $24 \pm 7.21$ & $1.33 \pm 2.30$ & $0 \pm 0$ & $0 \pm 0$ \\
120 & $3283.33 \pm 144.68$ & $1608 \pm 103.59$ & $28.66 \pm 5.68$ & $0.66 \pm 1.15$ & $0 \pm 0$ & $0 \pm 0$ \\
\hline
\end{tabular}

CT: Trichophyton rubrum without treatment with photosensitizer and light

L: Trichophyton rubrum + light

Hp IX + L+; Pp IX dimethyl ester + L+: commercial derivatives of protoporphyrin IX + Light

PpNpNI + L+; PpNpNA + L+: synthetic derivatives of protoporphyrin IX + Light 

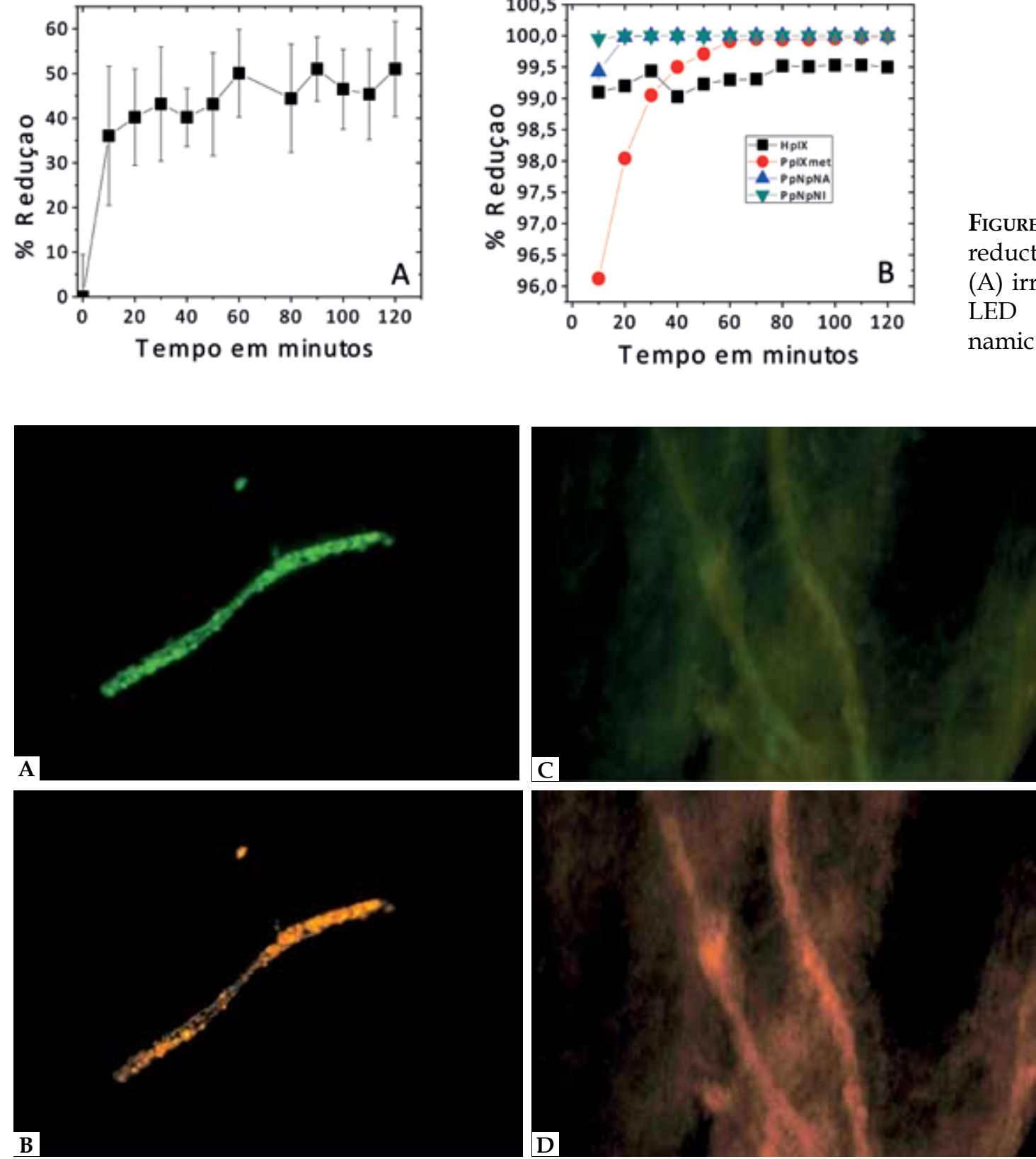

Figure 2: Percentage of reduction of $T$. rubrum (A) irradiated with blue LED (B) by photodynamic action
Figure 3:

Image $(\mathrm{A}, \mathrm{C})$ showing Trichophyton rubrum ATCC 28188 fluorescence, and $(B, D)$ image of fluorescence of the photosensitizers derivative from protoporphyrin IX incorporated in Trichophyton rubrum ATCC 28188. Vizualized in fluorescence microscope Zeiss Axiovert 200, using 100X objective lens
Figure 2A shows the percentage of reduction in CFUs of T. rubrum by irradiation at $400 \mathrm{~nm}$. In this assay, a 35\% reduction of CFU of T. rubrum was observed for the first irradiation cycle. This decrease was intensified for the first 6 cycles, presenting a plateau from the sixth cycle, in which there was a $50 \%$ reduction $\left(\mathrm{IC}_{50}\right)$. For hiher times, significant differences between the sixth and the following cycles were not observed.

Incorporation of PS in T. rubrum was observed by fluorescence microscopy, in which fluorescence was observed in red $(\approx 660 \mathrm{~nm})$, referring to emission of Pp IX derivatives. This emission was observed in hyphae and conidia, being perfectly differentiated from natural fluorescence of the species, which emits $450 \mathrm{~nm}$ when irradiated in ultraviolet. ${ }^{17}$ Figure 3 shows the fluorescence by emission of endogenous fluorophores and $\mathrm{Pp}$ IX derivatives incorporated in T. rubrum hyphae.

\section{DISCUSSION}

Microorganisms reduction by irradiation is widely known. ${ }^{18}$ The species may be eliminated by ultraviolet irradiation, classic disinfection method, since irradiation in these levels of energy leads to destruction of biomolecules by rupture of chemical bonds. ${ }^{19}$ 
However, energy emitted at $400 \mathrm{~nm}$ is not considered sufficient to provide this degradation process in biomolecules. Accordingly, the destruction of biological material by irradiation at $400 \mathrm{~nm}$ is attributed to photosensitization process by the presence of endogenous PS. For the tests, in which T. rubrum was irradiated in the presence of PS, we observed a reduction ratio higher than $96 \%$ of CFU for all tested PS.

In the assays using hematoporphyrin XI (HP IX), a classic PS, a 99\% reduction of CFU from the first irradiation cycle was observed. This activity had a positive performance for the first 3 cycles, remaining stable for the following clycles, thus indicating that the compound reached the plateau without achieving the complete elimination of CFUs of T. rubrum. This limitation may be attributed to a possible saturation of the membrane. ${ }^{20}$ Protoporphyrin dimethyl ester (Pp dimethyl ester IX) was the PS with lower reduction rate for the first cycle. However, a progressive evolution was observed over time, achieving, asymptotically, total reduction. It was observed that, after the third cycle, occurred a reduction greater than that presented for Hp IX. This superiority was attributed to the incorporation in the membrane, followed by enzymatic hydrolysis of the ester groups. ${ }^{21}$ This process provides improved internalization of the photosensitizer, whereas, in ester form, it presents greater interaction with lipophilic systems. Thus, after PS reaches the membrane, it will undergo hydrolysis, being conducted to the interior of the cells, resulting in better integration and consequently higher activity over time. ${ }^{22}$

PpNpNA and PpNpNI compounds presented greater activity (Figure 2). PpNpNA showed complete elimination of T. rubrum CFUs from the third irradiation cycle, while for PpNpNI, total elimination occurred from the second cycle. The superior activity of these compounds was attributed to the amphiphilic character and to the asymmetry. ${ }^{16}$ These characteristics enable the compounds to cross the membrane and internalize in the microorganisms, as observed in HeLas cells. In addition to the amphiphilicity and asymmetry, PpNpNA presents two groups of tertiary amine with $\mathrm{pKa} \approx 10$ on a same face of the molecule. Thus, it is expected that these compounds are partially protonated and attracted by biomolecules that have negative charges, by electrostatic forces. ${ }^{16} \mathrm{But} \mathrm{PpNpNI}$ is a permanent di-cationic, permanently susceptible to electrostatic attraction with anionic structures at any level of $\mathrm{pH}$.
When comparing PS structures, it is observed that protoporphyrin IX was changed only in their peripheral groups, not undergoing changes in the chromophore and, consequently, not undergoing changes in their photophysical properties. Accordingly, changes enabled the incorporation of PS, keeping the photooxidative potential of derivatives and, consequently, T. rubrum activity. In figure 3, we can observe, by the chhromophore fluorescence, that the compounds were perfectly incorporated, resulting in photooxidative damage when irradiated at $400 \mathrm{~nm}$, region where these compounds present the band with the highest absorption intensity, the Soret band.

Fluorescence at $660 \mathrm{~nm}$ proves the incorporation and internalization of PS in T. rubrum and shows that the elimination of the species occurred due to photoinduced process triggered near the chromophore, since these processes are initiated by protons or electrons tranference, radical reactions or electrophilic attack of singlet oxygen to membranes. ${ }^{23}$ Accordingly, it is necessary that the physicochemical properties of the photosensitizer and, consequently, its biodistribution, favors the incorporation in the parasite rather than in the host. ${ }^{24,25}$

Among the tested compounds, PpNpNA and PpNpNI were those that provided a greater incorporation and thus a higher activity in T. rubrum by affinity with biomolecules that have negative charges, such as glucosamine, glycoproteins and polysaccharides. ${ }^{26}$

\section{CONCLUSION}

The studies presented show that the photodynamic activity on Trichophyton rubrum relates to an adequate incorporation of PS, which can be made possible by changes in the peripheral functional groups of the chromophore. This strategy eliminates the need for a incorporation vehicle. Esterification of Pp IX enables the incorporation by lipophilicity and prolongs the activity, due to the controlled hydrolysis process of ester groups. Moreover, amphiphilic and cationic strategy adopted in PS PpNpNI and PpNpNA enables greater incorporation in shorter time, maximizing the treatment by reducing the period of exposure to irradiation and lower therapeutic doses, and the di-quaternary ammonium (PpNpNI) compound presents more effective by being cationic at all $\mathrm{pH}$ levels. $\square$ 


\section{REFERENCES}

1. Farias MR, Condas LAZ, Ramalho F, Bier D, Muro MD, Pimpão CT. Avaliação do estado de carreador assintomático de fungos dermatofiticos em felinos (Felis catus- Linnaeus, 1793) destinados à doação em centros de controle de zoonoses e sociedades protetoras de animais. Vet Zootec. 2011;18:306-12.

2. Quinn PJ, Markey BK, Leonard FC, Fitzpatrick ES, Fanning S, Hartigan PJ. Veterinary microbiology and microbial disease. Can Vet J. 2003;44:986.

3. Tortora GJ, Funke BR, Case CL. Microbiologia. 10 ed. Porto Alegre: Artmed; 2012. p.934.

4. Omero C, Dror Y, Freeman A. Trichoderma spp. antagonism to the dermatophyte Trichophyton rubrum: implications in treatment of onychomycosis. Mycopathologia. 2004;158:173-80

5. Smijs TG, Bouwstra JA, Talebi M, Pavel S. Investigation of conditions involved in the susceptibility of the dermatophyte Trichophyton rubrum to photodynamic treatment. J Antimicrob Chemother. 2007;60:750-9.

6. Peres NT, Maranhão FC, Rossi A, Martinez-Rossi NM. Dermatophytes: hostpathogen interaction and antifungal resistance. An Bras Dermatol. 2010;85:657-67.

7. Nir-Paz R, Elinav H, Pierard GE, Walker D, Maly A, Shapiro M, et al. Deep infection by Trichophyton rubrum in an immunocompromised patient. J Clin Microbiol. 2003;41:5298-301.

8. Maranhão FC, Paião FG, Fachin AL, Martinez-Rossi NM. Membrane transporter proteins are involved in Trichophyton rubrum pathogenesis. J Med Microbiol. 2009;58:163-8.

9. Borgers $M$, Degreef $H$, Cauwenbergh $G$. Fungal infections of the skin: infection process and antimycotic therapy. Curr Drug Targets. 2005;6:849-62.

10. Girois SB, Chapuis F, Decullier E, Revol BG. Adverse effects of antifungal therapies in invasive fungal infections: review and meta-analysis. Eur J Clin Microbiol Infect Dis. 2006;25:138-49.

11. Rodrigues GB, Ferreira LK, Wainwright M, Braga GU. Susceptibilities of the dermatophytes Trichophyton mentagrophytes and T. rubrum microconidia to photodynamic antimicrobial chemotherapy with novel phenothiazinium photosensitizers and red light. J Photochem Photobiol B. 2012;116:89-94.

12. Raab 0. Action of fluorescent materials on infusorial substances. Z Biol. 1900;39:524-46

13. Pereira AL, Pita JR. Alexander Fleming (1881-1955): da descoberta da penicilina (1928) ao prêmio Nobel (1945). Rev FLUP. 2005;6:129-51.

14. Vural E, Winfield HL, Shingleton AW, Horn TD, Shafirstein G. The effects of laser irradiation on Trichophyton rubrum growth. Lasers Med Sci. 2008;23:349-53.

15. Becker C, Bershow A. Lasers and photodynamic therapy in the treatment of onychomycosis: a review of the literature. Dermatol Online J. 2013;19:19611.

16. Uchoa AF, Oliveira CS, Baptista MS. Relationship between structure and photoactivity of porphyrins derived from protoporphyrin IX. J Porphyrins Phthalocyanines. 2010;14:832-45.

17. Gupta AK, Zaman M, Singh J. Diagnosis of Trichophyton rubrum from onychomycotic nail samples using polymerase chain reaction and calcofluor white microscopy. J Am Podiatr Med Assoc. 2008;98:224-8.

18. Yoshimura M, Namura S, Akamatsu H, Horio T. Antimicrobial effects of phototherapy and photochemotherapy in vivo and in vitro. $\mathrm{Br} \mathrm{J}$ Dermatol. 1996;135:528-32

19. Boyce RP, Howard-Flanders P. Genetic control of DNA breakdown and repair in $\mathrm{E}$. Coli $\mathrm{K}-12$ treated with mitomycin $\mathrm{C}$ or ultraviolet light. Z Vererbungsl. 1964;95:345-50

20. Santus R, Kohen C, Kohen E, Reyftmann JP, Morliere P, Dubertret L, et al. Permeation of lysosomal membranes in the course of photosensitization with methylene blue and hematoporphyrin: study by cellular microspectrofluorometry. Photochem Photobiol. 1983;38:71-7.

21. Eigen M. Proton Transfer, Acid-base Catalysis, and Enzymatic Hydrolysis. PART I: Elementary processes. Angewandte Chemi. 1964;3:1-19.

22. Bermúdez Moretti M, Correa García S, Perotti C, Batlle A, Casas A. DeltaAminolevulinic acid transport in murine mammary adenocarcinoma cells is mediated by beta transporters. Br J Cancer. 2002;87:471-4.
23. Weber G, Charitat T, Baptista MS, Uchoa AF, Pavani C, Junqueira HC, et al. Lipid oxidation induces structural changes in biomimetic membrane. Soft Matter. 2014;10:4241-7.

24. Kessel D, Woodburn k. Biodistribution of photosensitizing agentes. Int J Biochem. 1993;25:1377-83.

25. Song D, Lindoso JA, Oyafuso LK, Kanashiro EH, Cardoso JL, Uchoa AF, et al. Photodynamic therapy using methylene blue to treat cutaneous leishmaniasis. Photomed Laser Surg. 2011;29:711-5

26. Giavasis I. Bioactive fungal polysaccharides as potential functional ingredients in food and nutraceuticals. Curr Opin Biotechnol. 2014;26:162-73.
MAILING ADDRESS:
Rogério Rodrigo Ramos
Estrada Projetada F1 s/n
Fazenda Sta Rita,
15600-000 Fernandópolis, SP.
Cidade Universitária
05508-900 - São Paulo - SP
Brazil
Email: rogerio_enfer@hotmail.com

How to cite this article: Ramos RR, Kozusny-Andreani DI, Fernandes AU, Baptista MS. Photodinamic action of protoporphyrin IX derivatives on Trichophyton rubrum. An Bras Dermatol. 2016;91(2):135-40. 